\title{
On the definition of a kinetic equilibrium in global gyrokinetic simulations
}

\author{
P. Angelino \\ Ecole Polytechnique Fédérale de Lausanne (EPFL), Centre de Recherches en Physique des Plasmas, \\ Association Euratom Confédération Suisse, CH-1015, Lausanne, Switzerland
}

\author{
A. Bottino \\ Max-Planck Institut für Plasmaphysik, IPP-EURATOM Association, Garching, Germany \\ R. Hatzky \\ Computer Center of the Max-Planck-Gesellschaft and the Institute for Plasma Physics, D-85748 Garching, \\ Germany \\ S. Jolliet, O. Sauter, T. M. Tran, and L. Villard \\ Ecole Polytechnique Fédérale de Lausanne (EPFL), Centre de Recherches en Physique des Plasmas, \\ Association Euratom Confédération Suisse, CH-1015, Lausanne, Switzerland
}

(Received 28 November 2005; accepted 17 March 2006; published online 12 May 2006)

\begin{abstract}
Nonlinear electrostatic global gyrokinetic simulations of collisionless ion temperature gradient (ITG) turbulence and $E \times B$ zonal flows in axisymmetric toroidal plasmas are examined for different choices of the initial distribution function. Using a local Maxwellian leads to the generation of axisymmetric $E \times B$ flows that can be so strong as to prevent ITG mode growth. A method using a canonical Maxwellian is shown to avoid this spurious generation of $E \times B$ flows. In addition, a revised $\delta f$ scheme is introduced and compared to the standard $\delta f$ method. (C) 2006 American Institute of Physics. [DOI: 10.1063/1.2193947]
\end{abstract}

\section{INTRODUCTION}

In this paper we address the physical problem of defining a gyrokinetic equilibrium in a model where collisions are neglected. The general context is that of gyrokinetic simulations of turbulence generated by microinstabilities, such as ion temperature gradient (ITG) modes. The prominent role of axisymmetric $E \times B$ zonal flows, generated by turbulent fluctuations and having a regulating role for these, has been widely recognized. ${ }^{1}$ Hence the importance of avoiding spurious effects on zonal flows. In Ref. 2 it was shown that an inaccurate description of the gyrokinetic equilibrium can yield to unphysical excitation of zonal flow oscillations, with a measurable impact on the predicted heat flux. In collisionless systems, the gyrokinetic equilibrium distribution function $f_{\text {eq }}$ is defined to be constant along unperturbed orbits:

$$
\left.\frac{\mathrm{d} f_{\mathrm{eq}}}{\mathrm{d} t}\right|_{0}=0
$$

where the subscript 0 refers to the ordering with respect to the perturbation. In toroidal axisymmetric systems, the unperturbed orbits are characterized by three constants of motion: the particle energy: $\epsilon$, the magnetic moment $\mu$, and the toroidal canonical momentum $\psi_{0}$. Writing the equilibrium magnetic field as

$$
\mathbf{B}=F \nabla \varphi+\nabla \psi \times \nabla \varphi
$$

where $F=F(\psi)$ is the poloidal current flux function, $\psi$ is the poloidal magnetic flux, and $\varphi$ is the toroidal angle, the toroidal canonical momentum can be expressed as

$$
\psi_{0}=\psi+\frac{q_{i}}{m_{i}} \frac{F}{B} v_{\|}
$$

for species of charge $q_{i}$ and mass $m_{i}$. This implies that any equilibrium $f_{\text {eq }}$ (in the gyrokinetic sense) must be expressed in terms of $\epsilon, \mu$, and $\psi_{0}$. We shall call these $f_{\mathrm{C}}\left(\psi_{0}, \epsilon, \mu\right)$ canonical distribution functions. Often, an approximation is chosen for $f_{\text {eq }}$, in which $f_{\text {eq }}$ is specified in terms of $\psi$ instead of $\psi_{0}$. We shall refer to these as local distribution functions $f_{\mathrm{L}}(\psi, \epsilon, \mu)$. The difference between $\psi$ and $\psi_{0}$ is due to the radial orbit excursion of the gyrocenters. The error made by the local approximation to the true $f_{\mathrm{eq}}$ is therefore of the order of the ratio of the radial orbit width over the equilibrium gradient lengths of density $L_{n}$ or temperature $L_{T}$. For a local distribution function, Eq. (1) is not satisfied.

The $\delta f$ particle in cell (PIC) scheme ${ }^{3}$ is widely used to solve the gyrokinetic equations. The method, which we shall call standard- $\delta f$ in the following, is based on local Maxwellian equilibrium functions and a time evolution equation for $\delta f$, which is derived, neglecting the term $\mathrm{d} f_{\mathrm{L}} /\left.\mathrm{d} t\right|_{0} \neq 0$. This approximation cannot be justified in a general toroidal configuration. However, removing this approximation has the effect of driving spurious zonal flow oscillations. ${ }^{2}$ This undesirable effect seems to be avoided by the use of canonical equilibrium distribution functions.

In this paper, we will show that the specification of a canonical distribution function must avoid two problems. First, the symmetry in $v_{\|}$could be broken, leading to net parallel flows. Second, since ions and electrons have different orbit widths, it is not obvious to satisfy the equilibrium quasineutrality $\left(n_{0 i}=n_{0 e}\right)$ without introducing strong radial electric fields. These problems can lead to the unphysical suppression of the ITG instabilities through the spurious gen- 
eration of parallel and axisymmetric $E \times B$ flows, respectively. Here, we emphasize that the problem is not bound to the numerical method actually chosen to solve the equations, but it is a physical problem inherent to any gyrokinetic model where collisions are neglected. The problem of finding a gyrokinetic equilibrium, which satisfies both the absence of parallel flows and the quasineutrality condition in the collisionless limit, is not a trivial one. We propose a scheme that minimizes the $v_{\|}$asymmetry and the difference between $n_{0 i}$ and $n_{0 e}$, thus avoiding spurious flow drive.

In order to show that the problem is not specific to the particular method chosen to solve the gyrokinetic equations, we apply a modified algorithm based directly on the constancy of the full distribution function $f$ along the (perturbed) orbits. This scheme has been called direct- $\delta f{ }^{4}$ Here, it is applied to toroidal configurations and in the Appendix we show that it is equivalent to the standard- $\delta f$ scheme modified to include the term $\mathrm{d} f_{\mathrm{L}} /\left.\mathrm{d} t\right|_{0}$. Both schemes are also equivalent when a canonical $f_{\text {eq }}$ is used.

The numerical results presented in this paper are obtained with the code ORB5. ${ }^{5}$ The physical model implemented in the code ORB5 is the gyrokinetic Vlasov-Poisson system of equations as derived by Hahm. ${ }^{6}$ This integrodifferential system of equations describes electrostatic perturbations in a magnetized plasma. It aims at studying the electrostatic microinstabilities that are held responsible for enhanced heat transport in fusion plasmas. The system couples the gyrokinetic Vlasov equation for the ion distribution function to the Poisson equation. In the long wavelength limit, the latter reduces to the linearized quasi neutrality equation. A Boltzmann approximation is used for the electrons, which are supposed to react adiabatically to the electrostatic perturbation. For a complete and extensive introduction to the gyrokinetic model as it is implemented in ORB5 we refer to Hatzky et $a l .{ }^{7}$ where the model is applied to the $\theta$-pinch geometry. In the present paper, a general axisymmetric geometry is considered, and the code can run on ideal MHD equilibria. For this purpose, an interface with the equilibrium code $\mathrm{CHEASE}^{8}$ is included. The ion distribution function is discretized according to a particle-in-cell scheme (PIC) while the quasi neutrality equation is solved with a finite element method (FEM). Details on the discretization can be found in Ref. 7.

\section{SIMULATION PARAMETERS}

Two magnetic configurations are used for the simulations presented in this paper. The first configuration is the one reconstructed from a shot of the tokamak TCV, therefore it will be referred in the text as the TCV case. It is an elongated D-shaped plasma, with inverse aspect ratio $\epsilon \equiv a / R$ $=0.27$, elongation $\kappa=1.5, a=0.24 \mathrm{~m}=40 \rho_{i}\left(\rho_{i}\right.$ being the ion Larmor radius). The value of the magnetic field on axis is $B_{0}=1.44 \mathrm{~T}$. The ion temperature profile is obtained by integration of a gradient, given as

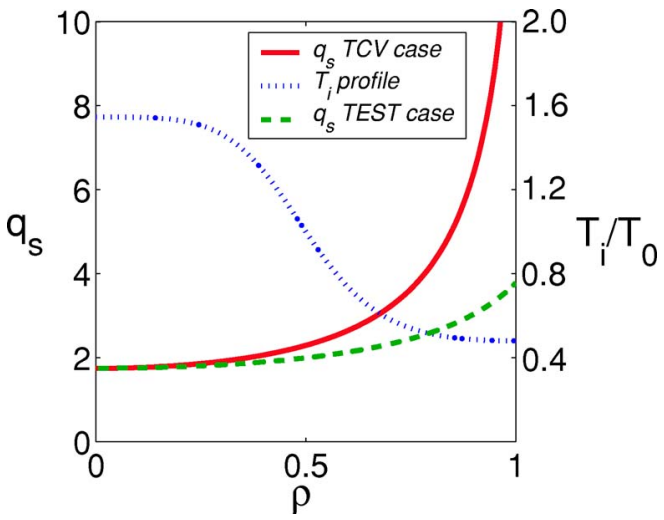

FIG. 1. (Color online) Safety factor $q_{s}$ profile for the $T C V$ case (solid line) and for the TEST case (dashed line). The ion temperature $T_{i}$ profile is also plotted (dotted line). The ion temperature is normalized to the value $T_{0} \equiv T(\rho=0.5)$.

$$
\begin{aligned}
\frac{\mathrm{d} T_{i}}{\mathrm{~d} \rho}= & -\frac{k_{T}}{1-\operatorname{sech}^{2}\left(\rho_{0} / \sigma_{s}\right)}\left[\operatorname{sech}^{2}\left(\frac{\rho-\rho_{0}}{\sigma_{s}}\right)\right. \\
& \left.-\operatorname{sech}^{2}\left(\rho_{0} / \sigma_{s}\right)\right],
\end{aligned}
$$

where $\rho=\sqrt{\psi / \psi_{a}}, \psi$ is the poloidal magnetic flux, and $\psi_{a}$ is its value at the plasma boundary, $\rho_{0}=0.5, k_{T}=3.0, \sigma_{s}=0.208$. The safety factor $q_{s}$ profile, and the temperature profile are shown in Fig. 1. The electron temperature profile is flat, with $T_{e}=T_{i}(\rho=0.5) \sim 1.8 \mathrm{keV}$, and the input density $n_{i 0}=n_{e 0}$ is constant. The second magnetic configuration is a circular plasma, with inverse aspect ratio $\epsilon \equiv a / R=0.2, a=0.18 \mathrm{~m}$ $=20 \rho_{i}$, and it will be referred to as TEST case. The value of the magnetic field on axis is $B_{0}=1.44 \mathrm{~T}$. The ion temperature profile has the same parameters as the TCV case. The $q_{s}$ and $T_{i}$ profiles are shown in Fig. 1. The electron temperature profile is flat, with $T_{e}=T_{i}(\rho=0.5) \sim 4 \mathrm{keV}$, and the input density $n_{i 0}=n_{e 0}$ is constant.

In the results, the physical quantities are expressed in normalized units. Temperatures and densities are normalized to their value at $\rho=0.5, T_{0}$ and $n_{0}$, respectively. The velocities are normalized to the sound speed $c_{s}$. The energy $E$ is given as $E /(n V T)$ and the heat flux $Q$ as $Q /\left[\langle n\rangle c_{s} T\right]$, where $n$ is the density and $\langle n\rangle$ its volume averaged value, $V$ is the plasma volume and $T=T_{0}$ is the electron temperature at $\rho=0.5$. The time is expressed in units of the inverse ion cyclotron frequency $\Omega_{c i}^{-1}$.

\section{INITIAL DISTRIBUTION FUNCTION}

In the framework of a control variate $\delta f$ method, we distinguish among the initial distribution function $f_{\text {init }}$, the equilibrium distribution function $f_{\text {eq }}$ and the control variate function $f_{0}$ (for a detailed definition and an exhaustive introduction to the control variate $\delta f$ method, we refer to the Appendix). The choice of $f_{\text {eq }}$ is constrained by physical equilibrium considerations. The choice of $f_{\text {init }}$ defines the initial condition of the system and could, in principle, be chosen arbitrarily. Since we are interested in the spontaneous evolution of the system from an equilibrium state to a turbulent state, $f_{\text {init }}$ is typically chosen very close to $f_{\text {eq. }}$. The choice of 


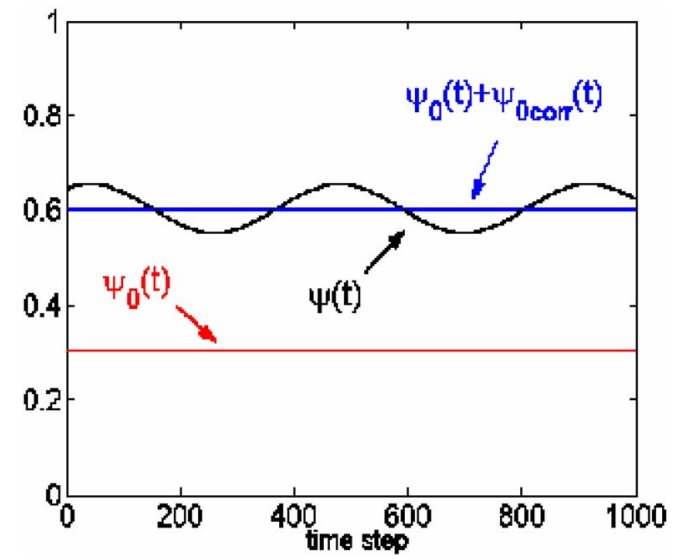

FIG. 2. (Color online) $\psi, \psi_{0}$, and $\psi_{0 \text { corr }}$ as a function of time for a given tracer on unperturbed trajectories. Output from ORB5.

$f_{0}$ is independent from the other functions, and it is used to minimize the numerical noise. At the beginning of a simulation, one can suppose the system is in a local thermodynamic equilibrium, and the natural choice for $f_{\text {init }}$ is a local Maxwellian:

$$
f_{\mathrm{LM}}(\psi, \epsilon, \mu)=\frac{n_{0}(\psi)}{(2 \pi)^{3 / 2} v_{\mathrm{th} i}^{3}(\psi)} \exp \left(-\frac{\epsilon}{v_{\mathrm{th} i}^{2}(\psi)}\right),
$$

$\psi$ being the poloidal flux, $\epsilon$ the particle energy per unit mass, $\mu$ the magnetic moment, $v_{\text {th } i}$ and $n_{0}$ initial thermal velocity and density profiles. But, collisions are not included in our model, and $f_{\mathrm{LM}}$ is not a kinetic equilibrium in a toroidal configuration (it is not constant along unperturbed particle trajectories). A more logical choice can be an $f_{\text {init }}$, function only of the constants of motion. ${ }^{2}$ So we have the canonical Maxwellian:

$$
f_{\mathrm{CM}}\left(\psi_{0}, \epsilon, \mu\right)=\frac{n_{0}\left(\psi_{0}\right)}{(2 \pi)^{3 / 2} v_{\mathrm{th} i}^{3}\left(\psi_{0}\right)} \exp \left(-\frac{\epsilon}{v_{\mathrm{th} i}^{2}\left(\psi_{0}\right)}\right),
$$

where the toroidal canonical momentum $\psi_{0}$, is now constant on the unperturbed characteristics. Figure 2 shows $\psi$ and $\psi_{0}$ on an unperturbed trajectory as function of time. $\psi$ oscillates around a mean value, $\psi_{0}$ is constant, but its value is far away from the mean value of $\psi$. This means that the effective temperature and density profiles (evaluated from the particle distribution function, after the loading with $f_{\mathrm{CM}}$ ) differ substantially from the input ones given as a function of $\psi$, as shown in Fig. 3. The canonical Maxwellian can lead to large parallel flows. Depending on the configuration, particularly for small system size and large $n_{0}$ and $v_{\text {th } i}$ gradients, they can be so strong as not to allow the instability to develop. But, if we add to $\psi_{0}$ a function of the constants of motion, we obtain a $\hat{\psi}=\psi_{0}+\psi_{0 \text { corr }}$ that is still a constant of motion. We can use this degree of freedom to reduce the gap between the mean value of $\psi$ and $\hat{\psi}$. Thus, we define

$$
\psi_{0 \mathrm{corr}} \equiv-\operatorname{sign}\left(v_{\|}\right) \frac{q_{i}}{m_{i}} R_{0} \sqrt{2\left(\epsilon-\mu B_{\mathrm{mag}}\right)} \mathcal{H}\left(\epsilon-\mu B_{\mathrm{mag}}\right)
$$

which is an estimate of the average radial excursion of the particle orbit. $B_{\text {mag }}$ is the magnetic field on magnetic axis $(\mathcal{H}$
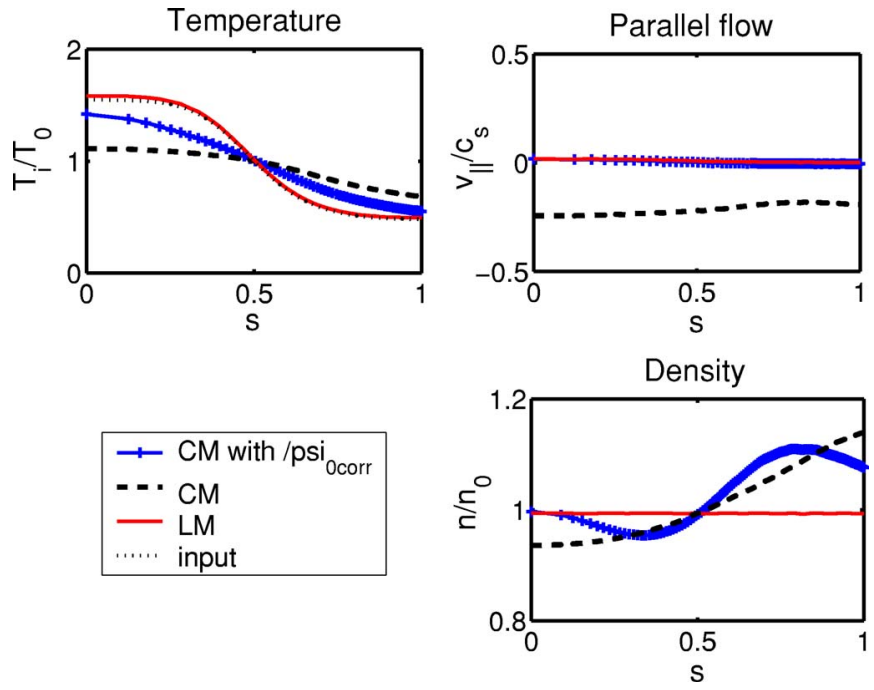

FIG. 3. (Color online) Effective initial profiles for the TEST case. Effective temperature, density and parallel flow are plotted as a function of the normalized radius $s=\rho / a$. The different curves on each plot represents results from runs with different initial distribution functions: Canonical Maxwellian (CM), local Maxwellian and canonical Maxwellian with correction to $\psi_{0}$.

is the Heaviside function). With $f_{\text {init }}=f_{\mathrm{CM}}(\hat{\psi}, \epsilon, \mu), \hat{\psi}$ is now close to the mean value of $\psi$ (Fig. 2), the profiles are close to the local ones (Fig. 3), and the initial large parallel flows are reduced to a level compatible with the development of the instability.

\section{SPURIOUS GENERATION OF AXISYMMETRIC FIELDS WITH LOCAL MAXWELLIAN}

In the standard- $\delta f$ method, $f_{0}=f_{\text {init }}$ is supposed to be an equilibrium function and Eq. (A11) is applied. If $f_{0}$ is chosen to be a local Maxwellian, which is not an exact solution of the unperturbed equation of motion, Eq. (A12) must be applied. Thus we have an extra term in the $\delta f$ evolution equation:

$$
-\left.\frac{\mathrm{d} f_{0}}{\mathrm{~d} t}\right|_{0}=-\left.\frac{\partial f_{0}}{\partial \mu} \frac{\mathrm{d} \mu}{\mathrm{d} t}\right|_{0}-\left.\frac{\partial f_{0}}{\partial \epsilon} \frac{\mathrm{d} \epsilon}{\mathrm{d} t}\right|_{0}-\left.\frac{\partial f_{0}}{\partial \psi} \frac{\mathrm{d} \psi}{\mathrm{d} t}\right|_{0} .
$$

Since $\mu$ and $\epsilon$ are constants of motion,

$$
-\left.\frac{\mathrm{d} f_{0}}{\mathrm{~d} t}\right|_{0}=-\left.\frac{\partial f_{0}}{\partial \psi} \nabla \psi \cdot \frac{\mathrm{d} \mathbf{R}}{\mathrm{d} t}\right|_{0},
$$

with the motion along the unperturbed trajectories described by

$$
\left.\frac{\mathrm{d} \mathbf{R}}{\mathrm{d} t}\right|_{0}=v_{\|} \mathbf{h}+\mathbf{v}_{\nabla b}+\mathbf{v}_{\nabla P}
$$

The three terms on the right hand side represent the parallel motion along the magnetic field lines $(\mathbf{h}=\mathbf{B} / B)$, the magnetic and pressure gradient drifts, respectively. Reminding that $\boldsymbol{\nabla} \psi \cdot \mathbf{h}=0$, 


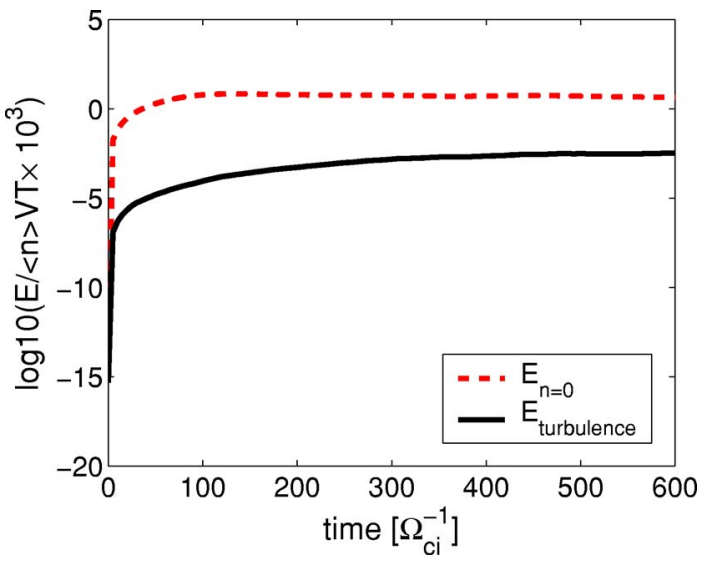

FIG. 4. (Color online) Energy of the $n=0$ mode (dashed line) and of the turbulence (solid line) as a function of time. Results from the TEST case with a standard- $\delta f$ scheme, the local Maxwellian as an equilibrium function and the $\mathrm{d} f_{0} /\left.\mathrm{d} t\right|_{0}$ term included in the $\delta f$ equation. The strong radial electric field hinders the turbulent modes already in the early phase.

$$
\begin{aligned}
-\left.\frac{\mathrm{d} f_{0}}{\mathrm{~d} t}\right|_{0}= & -\frac{\partial f_{0}}{\partial \psi} \frac{1}{B_{\|}^{*}}\left(\frac{\mu B+v_{\|}^{2}}{\Omega_{c i}} \mathbf{h} \times \nabla B\right. \\
& \left.+\frac{v_{\|}^{2}}{\Omega_{c i}} \mathbf{h} \times \mu_{0} \frac{\nabla P}{B}\right) \cdot \nabla \psi,
\end{aligned}
$$

with:

$$
\begin{aligned}
& B_{\|}^{*}=B+\frac{m_{i}}{q_{i}} v_{\|} \mathbf{h} \cdot \nabla \times \mathbf{h}, \\
& \Omega_{c i}=\frac{q_{i}}{m_{i}} B .
\end{aligned}
$$

Since $\nabla P \| \nabla \psi$ :

$$
-\left.\frac{\mathrm{d} f_{0}}{\mathrm{~d} t}\right|_{0}=-\frac{\partial f_{0}}{\partial \psi} \frac{\mu B+v_{\|}^{2}}{B_{\|}^{*} \Omega_{i}} \mathbf{h} \times \nabla B \cdot \nabla \psi .
$$

This term relies only on equilibrium quantities and is responsible for the spurious zonal flow drive addressed by Idomura. $^{2}$ Zonal flows are axisymmetric $\mathbf{E} \times \mathbf{B}$ flows; they correspond to the radial $n=0, m=0$ component of the electric field (here, $n$ and $m$ are, respectively, the toroidal and poloidal Fourier mode number). Modes with $n=0$ are linearly stable, but they are excited by turbulence trough nonlinear coupling. ${ }^{9}$ They should not appear from the beginning of the simulation, but they should follow the development of the turbulence after the linear phase. As shown in Fig. 4, the inclusion of the term in Eq. (14) drives the zonal flows from the very beginning of the simulation to level orders of magnitude higher than the turbulence level. This effect is not of numerical origin, but is due to the choice of initial conditions, i.e., the system is not close to an equilibrium and, as a result of the particle magnetic drift across magnetic surfaces a strong radial electric field develops in order to maintain the quasineutrality imposed by the model. Following the usual practice in tokamak PIC simulations, we could neglect the term in Eq. (14) (thus incorrectly assuming that the local Maxwellian is a true equilibrium function) and we would get rid of this initial zonal flow (Fig. 5). But, dropping $\mathrm{d} f_{0} /\left.\mathrm{d} t\right|_{0}$

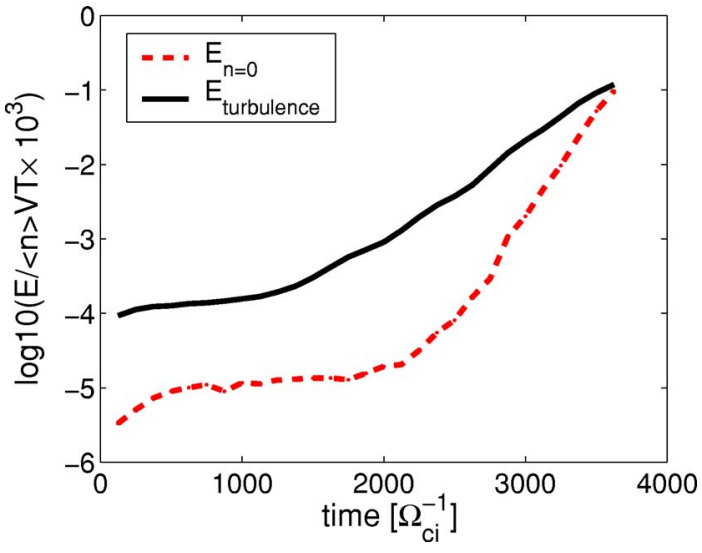

FIG. 5. (Color online) Energy of the $n=0$ mode (dashed line) and of the turbulence (solid line) as a function of time. Results from the TEST case with standard- $\delta f$ scheme, local Maxwellian as an initial function, and $n e-$ glecting the $\mathrm{d} f_{0} /\left.\mathrm{d} t\right|_{0}$ term in the $\delta f$ equation. The radial $n=0$ mode is linearly stable and the turbulence can develop freely.

cannot be justified in a perturbative framework because it is a zeroth order term. The direct- $\delta f$ scheme correctly solves the $\delta f$ equation and includes this term automatically, so with this scheme we get the same results as shown in Fig. 4.

\section{SPURIOUS GENERATION OF AXISYMMETRIC FIELDS WITH CANONICAL MAXWELLIAN}

To investigate the role played by a canonical Maxwell$i a n$ as an equilibrium distribution function, we have to analyze in detail the quasineutrality equation:

$$
\left\langle n_{i}\right\rangle(\mathbf{x}, t)+n_{\text {pol }}(\mathbf{x}, t) \approx n_{e}(\mathbf{x}, t),
$$

where $n_{e}$ is the electron density, $n_{\text {pol }}$ is the linearized polarization density, ${ }^{7}$ and $\left\langle n_{i}\right\rangle$, the gyroaveraged density, is defined as

$$
\left\langle n_{i}\right\rangle(\mathbf{x}, t) \equiv \int f(\mathbf{R}, \epsilon, \mu, t) \delta\left(\mathbf{R}+\rho_{i}-\mathbf{x}\right) \mathrm{d} \mathbf{R} \mathrm{d} \mathbf{v} ;
$$

here, $\rho_{i}$ is the ion gyroradius.

We suppose Boltzmann electrons. Electrons are in thermodynamic equilibrium on each flux surface, responding adiabatically to the electric field perturbation, hence their distribution function is a local Maxwellian and the density can be written as

$$
n_{e}(\mathbf{x}, t)=n_{0 e}(\psi) \exp [-C(\psi)] \exp \left(-\frac{e \phi}{k_{\mathrm{B}} T_{e}(\psi)}\right),
$$

$n_{0 e}$ is the electron initial density, $C(\psi)$ is a generic function of the poloidal flux $\psi, e$ the electron charge, $\phi$ the perturbed electrostatic potential, $T_{e}$ the electron temperature. We suppose the electron particle number on a flux surface should be constant. The electron density becomes

$$
n_{e}(\mathbf{x}, t) \approx n_{0 e}(\psi)+\frac{e n_{0 e}(\psi)}{k_{\mathrm{B}} T_{e}(\psi)}[\phi(\mathbf{x}, t)-\bar{\phi}(\psi, t)],
$$

where $\bar{\phi}$ is the flux surface averaged electrostatic potential: 


$$
\bar{\phi}(\psi, t)=\frac{\iint[\phi(\psi, \theta, \varphi, t) J(\psi, \theta)] \mathrm{d} \theta \mathrm{d} \varphi}{\iint J(\psi, \theta) \mathrm{d} \theta \mathrm{d} \varphi} .
$$

We have introduced the Jacobian $J$, which, for a axisymmetric system depends only on the poloidal flux $\psi$ and the poloidal coordinate $\theta$. With the $\delta f$ ansatz the gyroaveraged density becomes

$$
\begin{aligned}
\left\langle n_{i}\right\rangle(\mathbf{x}, t) & \equiv \int\left[f_{0}(\mathbf{R}, \epsilon, \mu)+\delta f(\mathbf{R}, \epsilon, \mu, t)\right] \delta\left(\mathbf{R}+\rho_{i}-\mathbf{x}\right) \mathrm{d} \mathbf{R} \mathrm{d} \mathbf{v} \\
& =\left\langle n_{0 i}\right\rangle+\int \delta f(\mathbf{R}, \epsilon, \mu, t) \delta\left(\mathbf{R}+\rho_{i}-\mathbf{x}\right) \mathrm{d} \mathbf{R} \mathrm{d} \mathbf{v} .
\end{aligned}
$$

If the ion distribution function is a local Maxwellian, approximating $\left\langle n_{0 i}\right\rangle \approx n_{0 i}$ (accordingly to the gyroordering), the electron and ion equilibrium densities cancel in the quasineutrality equation. If we choose a canonical Maxwellian for the ion distribution function, we have an extra term in the quasineutrality equation coming from the difference between ion and electron equilibrium densities, $\left\langle n_{0 i}\right\rangle \neq n_{0 e}(\psi)$. Indeed, the correct way to do it is to take a canonical Maxwellian equilibrium $f_{\mathrm{eq}}=f_{\mathrm{CM}}$, and a local Maxwellian control variate function $f_{0}=f_{\mathrm{LM}}$. Thus, the gyroaveraged ion density can be written as

$$
\begin{aligned}
\left\langle n_{i}\right\rangle(\mathbf{x}, t)= & \int f_{\mathrm{LM}} \delta\left(\mathbf{R}+\rho_{i}-\mathbf{x}\right) \mathrm{d} \mathbf{R} \mathrm{d} \mathbf{v}+\int\left[f_{\mathrm{CM}}-f_{\mathrm{LM}}\right] \\
& \times \delta\left(\mathbf{R}+\rho_{i}-\mathbf{x}\right) \mathrm{d} \mathbf{R} \mathrm{d} \mathbf{v} \\
& +\int \delta f \delta\left(\mathbf{R}+\rho_{i}-\mathbf{x}\right) \mathrm{d} \mathbf{R} \mathrm{d} \mathbf{v} .
\end{aligned}
$$

The first integral on the rhs cancels with $n_{0 e}$, and an extra term appears:

$$
\begin{aligned}
& \int\left(f_{\mathrm{CM}}-f_{\mathrm{LM}}\right) \delta\left(\mathbf{R}+\rho_{i}-\mathbf{x}\right) \mathrm{d} \mathbf{R} \mathrm{d} \mathbf{v} \approx n_{0 i}-n_{0 e} \\
& =n_{0}\left(\psi_{0}\right)-n_{0}(\psi) .
\end{aligned}
$$

The difference between electron and ion densities leads to the formation of a strong axisymmetric electric field. Our simulations (Fig. 6) show that it can be strong enough to prevent any unstable ITG mode to grow. We note that the $n=0$ field energy is of a comparable value to that created when using the local Maxwellian initial distribution (Fig. 4), which is due to the fact that in both cases the $n=0$ field is due to the difference between a local and a canonical Maxwellian. One could drop the term in Eq. (22)), thus approximating $f_{\mathrm{LM}}=f_{\mathrm{CM}}$. This could be justified in equilibrium configurations where $\psi_{0}$ is close to $\psi$ (large aspect ratio and low values of the safety factor). But, in general, it should be retained because it is a zeroth order correction and it leads to effects of the order of the inclusion of $\mathrm{d} f_{0} /\left.\mathrm{d} t\right|_{0}$ [Eq. (14)] in the $\delta f$ equation (compare Fig. 4 and Fig. 6).

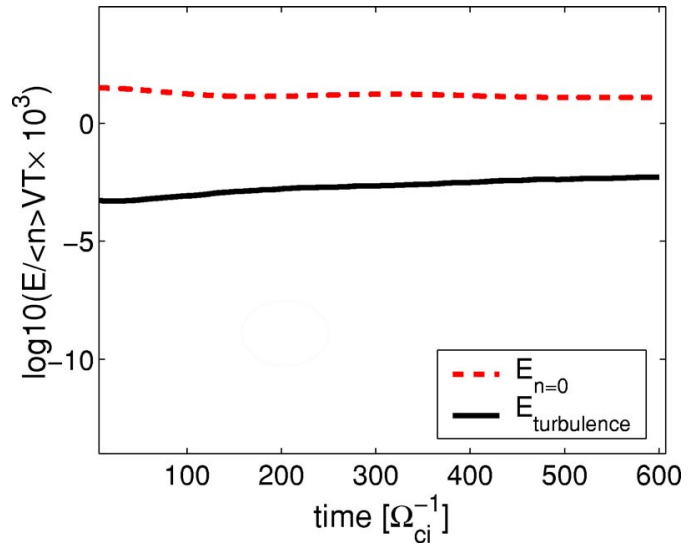

FIG. 6. (Color online) Energy of the $n=0$ mode (dotted line) and of the turbulence (solid line) as a function of time. Results from the TEST case with a direct- $\delta f$ scheme, the canonical Maxwellian as an equilibrium function, and the local Maxwellian as a control variate function. The strong radial electric field hinders the turbulent modes even in the early linear phase.

\section{ELIMINATION OF SPURIOUS FIELD GENERATION}

In the two previous sections we highlight the difficulties of setting up initial conditions that do not lead to an initial radial electric field so strong as to suppress completely the turbulence. In a collisionless model, the local Maxwellian is a thermodynamic equilibrium but is not a gyrokinetic equilibrium, so a strong electric field develops from the beginning of the simulation in order to enforce the quasineutrality. On the other side, the canonical Maxwellian, which is a gyrokinetic equilibrium, is not a solution of the quasineutrality equation with adiabatic electrons. In a quasineutrality equation where a fluid model for electrons and a kinetic model for ions coexist, adiabatic electrons cannot neutralize an ion charge density that depends also on the poloidal angle. ${ }^{11}$ Again, equilibrium electric fields develop to enforce the quasineutrality. Without including collisions, with the numerical difficulties involved for a PIC code, the only possibility is to choose a $n_{0 e}(\psi)$ such as to minimize Eq. (22). Therefore we introduce a new scheme where the electron distribution function $n_{0 e}$ is no longer evaluated from an analytical expression, but it has to be integrated from the ion distribution function after the particle loading:

$$
n_{0 e}(\psi)=\frac{1}{2 \pi} \int\left(\int f_{\mathrm{CM}} \delta\left(\mathbf{R}+\rho_{i}-\mathbf{x}\right) \mathrm{d} \mathbf{R} \mathrm{d} \mathbf{v}\right) \mathrm{d} \theta
$$

Thus, now we have

$$
n_{0 e}(\psi) \simeq\left\langle n_{0 i}\right\rangle \text {. }
$$

We remark that this equality cannot be exact, since the gyroaveraged ion density depends on the poloidal angle $\theta$, while, by definition, the electron density depends only on $\psi$. The approximation in Eq. (24) is equivalent to neglect banana orbit effects and it is consistent with the approximation in the polarization density. With this definition of the electron density, we are able to use a canonical Maxwellian as control variate function; therefore $f_{0}=f_{\mathrm{eq}}=f_{\mathrm{CM}}$, and Eq. (21) becomes 


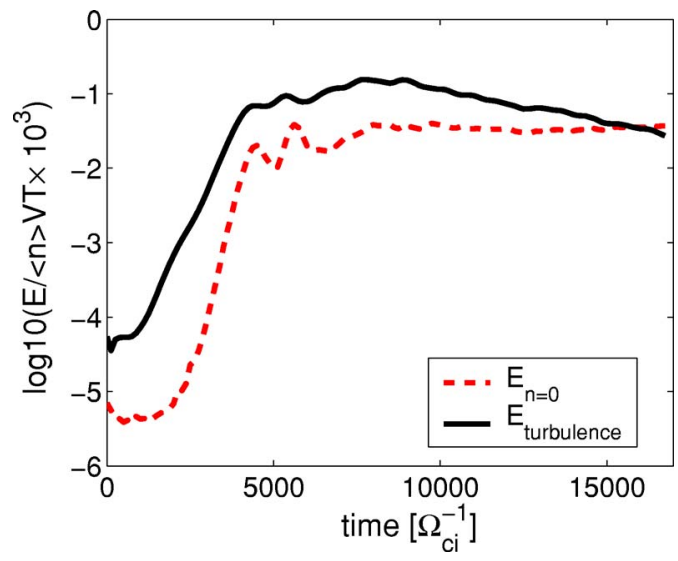

FIG. 7. (Color online) Energy of the $n=0$ mode (dotted line) and of the turbulence (solid line) as a function of time. Results from the TCV case with a direct- $\delta f$ scheme and canonical Maxwellian as anequilibrium function and the control variate function. The initial electron density $n_{0 e}(\psi)$ is obtained from direct integration of the drift-kinetic ion distribution function. No spurious axisymmetric electric field is present, and the turbulence develops freely.

$$
\begin{aligned}
\left\langle n_{i}\right\rangle(\mathbf{x}, t)= & \int f_{\mathrm{CM}} \delta\left(\mathbf{R}+\rho_{i}-\mathbf{x}\right) \mathrm{d} \mathbf{R} \mathrm{d} \mathbf{v} \\
& +\int \delta f \delta\left(\mathbf{R}+\rho_{i}-\mathbf{x}\right) \mathrm{d} \mathbf{R} \mathrm{d} \mathbf{v},
\end{aligned}
$$

which means that, now, the electron and ion equilibrium densities cancel out in the quasineutrality equation and the extra term [Eq. (22)] is not present anymore.

We implemented this integration in ORB5 with standard$\delta f$ and direct- $\delta f$ schemes and we succeeded in eliminating the spurious axisymmetric electric field in both method, as shown in Fig. 7 using the direct- $\delta f$ method (standard $\delta f$ gives identical results). Moreover, our simulations show that the gyroaveraging of the density is indeed a correction of higher order, and we obtain essentially the same results with a drift kinetic expression:

$$
n_{0 e}(\psi)=\frac{1}{2 \pi} \int f_{\mathrm{CM}} \mathrm{d} \mathbf{v} \mathrm{d} \varphi \mathrm{d} \theta .
$$

\section{CONCLUSIONS}

The choice of the equilibrium distribution function plays a major role in spurious axisymmetric field generation. When a local Maxwellian, $f_{\mathrm{LM}}(\psi, \epsilon, \mu)$, is chosen, which is not a true gyrokinetic equilibrium function, a strong electric field develops from the beginning of the simulation as particles depart from their initial magnetic surface due to magnetic drifts. The $n=0$ mode can be so large that the turbulence does not even start to develop. Dropping the $\mathrm{d} f_{0} /\left.\mathrm{d} t\right|_{0}$ in the $\delta f$ evolution equation is the only possible way to overcome this problem. But, the suppression of this term cannot be physically justified since it is a zeroth order term in Eq. (A10). This is the reason for introducing a canonical Maxwellian, $f_{\mathrm{CM}}\left(\psi_{0}, \epsilon, \mu\right)$. Since it is a gyrokinetic equilibrium distribution function, $\mathrm{d} f_{0} /\left.\mathrm{d} t\right|_{0}$ is null by construction. However, two problems arise from the use of the canonical Max- wellian. The first one is related to the effective temperature, density and shear parallel flow profiles. For certain equilibrium configurations (small system scale, strong gradients), they can assume values for which the turbulence is completely suppressed and therefore these configurations cannot be studied. The problem can be overcome introducing a correction to $\psi_{0}$, which is an estimate of the average radial excursion of the particle orbit. With this correction the effective profiles are close to the local ones. The second problem arises from the difference in the equilibrium functions of ions and electrons. This problem has been addressed in the present work for a hybrid theory with adiabatic electrons and kinetic ions, but it also affects any collisionless full kinetic model. The equilibrium function of electrons differs from the one of the ions and even without an initial perturbation, a quasineutrality $n_{0 i} \simeq n_{0 e}$ cannot be established without introducing a large initial axisymmetric electrostatic potential, which again suppresses the turbulence. In the framework of a hybrid theory, dropping the term representing the difference between $f_{\mathrm{LM}}$ and $f_{\mathrm{CM}}$ cannot be physically justified. We have found the solution to the above problems by constructing the electron equilibrium density, $n_{0 e}(\psi)$, by integration of the canonical distribution of ions. The choice of the initial distribution function is of major concern also for non axisymmetric three dimensional (3D) systems (such as stellarators or tokamaks with magnetic islands). In these configurations a canonical Maxwellian cannot be built, since $\psi_{0}$ is no more a constant of motion, and a collisionless gyrokinetic equilibrium with spatial density and temperature gradients cannot be found without equilibrium electric fields. Therefore, for these 3D systems, the problem is still an open question and should be approached within the frame of collisional (neoclassical) theory. Within a collisional model the local Maxwellian becomes closer to a true kinetic equilibrium and can be introduced as initial distribution function for both ions and electrons.

In conclusion, a direct- $\delta f$ or standard- $\delta f$ method with canonical Maxwellian plus correction term and integrated $n_{0 e}$, can be used to obtain physically meaningful simulations in toroidal geometry.

\section{ACKNOWLEDGMENTS}

This work has been partly supported by the Swiss National Science Foundation. The simulations have been run on the Pleiades cluster and IBM Blue Gene parallel machine at EPFL. We thank Dr. A. Könies, Dr. J. Graves, and Dr. Y. Idomura for stimulating discussions.

\section{APPENDIX A: THE CONTROL VARIATE METHOD AND THE DIRECT- $\delta f$ SCHEME}

The $\delta f$ method $^{3}$ is extensively used in gyrokinetic particle-in-cell (PIC) simulations of plasma turbulence to reduce the statistical noise associated with the PIC scheme. The standard implementation of this algorithm involves the integration of an evolution equation for $\delta f$. This equation is actually redundant, ${ }^{10}$ and a $\delta f$ scheme can be constructed without it. This revised $\delta f$ algorithm (in the following it will be referred to as direct- $\delta f$ scheme) was already successfully 
implemented in the $\theta$-pinch geometry. ${ }^{4}$ In this appendix, the implementation of the direct- $\delta f$ in a general toroidal geometry is introduced and compared to the standard $\delta f$ method.

Aydemir ${ }^{10}$ has shown, in the framework of Monte Carlo methods, that the $\delta f$ method is equivalent to a control variates method of variance reduction. In this scheme, the statistical noise, introduced with the PIC sampling of the distribution function, is reduced by splitting the distribution function $f(\mathbf{Z})$, where $\mathbf{Z}$ is a set of phase-space coordinates, in a readily calculable function $f_{0}(\mathbf{Z})$ and a statistically approximated part $\delta f(\mathbf{Z})$ :

$$
f(\mathbf{Z})=f_{0}(\mathbf{Z})+\delta f(\mathbf{Z}) .
$$

Typically, the solution of a given problem requires the evaluation of various moments of $f$ on the finite element (or B-spline) grid. In particular, the charge assignment, i.e., the charge density deposition on the finite element grid corresponds to the calculation of the order 0 momentum of $f$ :

$$
b_{\nu}=\int f(\mathbf{Z}) \Lambda_{\nu}(\mathbf{Z}) \mathrm{d} \Gamma,
$$

where $\Lambda_{\nu}(\mathbf{Z})$ are the finite element basis functions, and $\mathrm{d} \Gamma \equiv \mathcal{J} \mathrm{d} \mathbf{Z}$. $\mathcal{J}$ is the phase space Jacobian. A small error in the evaluation of $b_{\nu}$ can lead to a fast deterioration of the quality of the simulation. In a scheme where the full $f$ is statistically sampled, the standard error in the estimate of $b_{\nu}$ will be proportional to the square root of the variance $\left(\sigma_{g_{\nu}}\right)$ of the function ${ }^{4}$ :

$$
\begin{aligned}
& g_{\nu}=\frac{f(\mathbf{Z}) \Lambda_{\nu}(\mathbf{Z})}{p(\mathbf{Z})}, \\
& \sigma_{g_{\nu}}^{2}=\int\left[g_{\nu}(\mathbf{Z})-\left\langle g_{\nu}\right\rangle\right]^{2} p(\mathbf{Z}) \mathrm{d} \Gamma,
\end{aligned}
$$

where $p(\mathbf{Z})$ is a probability density function, the distribution of the sampling PIC markers. With the split in Eq. (A1), we can write

$$
b_{\nu}=\int f_{0}(\mathbf{Z}) \Lambda_{\nu}(\mathbf{Z}) \mathrm{d} \Gamma+\int \delta f(\mathbf{Z}) \Lambda_{\nu}(\mathbf{Z}) \mathrm{d} \Gamma .
$$

Since the evaluation of the first integral does not involve any statistical error, the standard error will now be proportional to the square root of the variance $\left(\sigma_{h_{v}}\right)$ of the function

$$
h_{\nu}=\frac{\delta f(\mathbf{Z}) \Lambda_{\nu}(\mathbf{Z})}{p(\mathbf{Z})} .
$$

Thus, the reduction of the error is of the order

$$
\frac{\sigma_{h_{v}}}{\sigma_{g_{\nu}}} \sim \frac{|\delta f|}{f} .
$$

This reduction of the error comes from the extra information we put in the scheme: the known function $f_{0}$ closely approximates $f$, i.e., $\left|f-f_{0}\right| \ll f$. In the standard $\delta f$ method, this extra information comes from the physical argument that $\delta f$ is a small perturbation around an equilibrium distribution $f_{\text {eq }}$, so we have

$$
f=f_{\mathrm{eq}}+\delta f \text {. }
$$

In fact, we want to stress that, in the control variates method, the choice of $f_{0}$ is not bound to the choice of an initial equilibrium function $f_{\text {eq }}$. It can be any given function, with the only requirement of being able to evaluate analytically or numerically momenta of $f_{0}$ without using sampling markers, i.e., without introducing numerical noise. Obviously the closer $f_{0}$ is to $f$, the smaller the statistical error is [Eq. (A7)]. Therefore $f_{0}$ can be evolved during the simulation to constantly approach the total $f$ when it moves away from $f_{\text {eq }}$ (adaptive $f_{0}$ ). Having given a general introduction to the control variates method, we can now turn to analyze how it is applied to the gyrokinetic Vlasov-Poisson system of equations. The full ion gyrocenter distribution function $f$ is constant along the characteristics of the gyrokinetic Vlasov equation. This statement can be used to find a time evolution equation for the statistically sampled part of the distribution function $\delta f$. We use the ansatz Eq. (A8), choosing $f_{0}=f_{\text {eq }}$. Particle trajectories are also split in an equilibrium part (where the electrostatic perturbation potential $\phi$ is set to zero) and a perturbed part,

$$
\frac{\mathrm{d} \mathbf{Z}_{i}}{\mathrm{~d} t}=\left.\frac{\mathrm{d} \mathbf{Z}_{i}}{\mathrm{~d} t}\right|_{0}+\left.\frac{\mathrm{d} \mathbf{Z}_{i}}{\mathrm{~d} t}\right|_{1} .
$$

From $\mathrm{d} f / \mathrm{d} t=0$ along the characteristics, we can write

$$
\frac{\mathrm{d}}{\mathrm{d} t} \delta f=-\frac{\mathrm{d} f_{\mathrm{eq}}}{\mathrm{d} t}=-\left.\frac{\mathrm{d} f_{\mathrm{eq}}}{\mathrm{d} t}\right|_{0}-\left.\frac{\mathrm{d} f_{\mathrm{eq}}}{\mathrm{d} t}\right|_{1} .
$$

Now, $f_{\text {eq }}$ is an exact solution of the unperturbed Vlasov equation $\mathrm{d} f_{\text {eq }} /\left.\mathrm{d} t\right|_{0}=0$, and the $\delta f$ evolution equation is

$$
\frac{\mathrm{d}}{\mathrm{d} t} \delta f=-\left.\frac{\mathrm{d} f_{\mathrm{eq}}}{\mathrm{d} t}\right|_{1} .
$$

We refer to this scheme, with a $\delta f$ evolution equation and where $f_{0}=f_{\text {eq }}$ is supposed to be a true equilibrium distribution function, as the standard- $\delta f$ scheme. More generally, in the control variate method, we can choose a $f_{0} \neq f_{\text {eq. }}$. But, if $f_{0}$ is not a true equilibrium distribution function, we get an extra term coming from the evolution of $f_{0}$ on the unperturbed characteristics in the $\delta f$ equation, which now is written as

$$
\frac{\mathrm{d}}{\mathrm{d} t} \delta f=-\left.\frac{\mathrm{d} f_{0}}{\mathrm{~d} t}\right|_{0}-\left.\frac{\mathrm{d} f_{0}}{\mathrm{~d} t}\right|_{1} .
$$

As pointed out by Aydemir ${ }^{10}$ this equation is actually redundant, $f\left[\mathbf{Z}_{i}(t)\right]=f\left[\mathbf{Z}_{i}\left(t_{0}\right)\right]$ and $\delta f$, at a given time $t$, can be evaluated from

$$
\delta f\left[\mathbf{Z}_{i}(t)\right]=f\left[\mathbf{Z}_{i}\left(t_{0}\right)\right]-f_{0}\left[\mathbf{Z}_{i}(t)\right] .
$$

We call this scheme the direct- $\delta f$ method.

The standard- $\delta f$ and direct- $\delta f$ schemes are theoretically equivalent. But, to get the same numerical results, the implementation must be done carefully, in particular, the evaluation of $f_{0}$ can be done in two different ways and lead to a divergence of the two methods. In the direct- $\delta f$ scheme, $f_{0}$ is needed in order to solve Eq. (A13). Being a known function, $f_{0}$ is sampled at the marker position $\mathbf{Z}_{i}(t)$, and the accuracy 

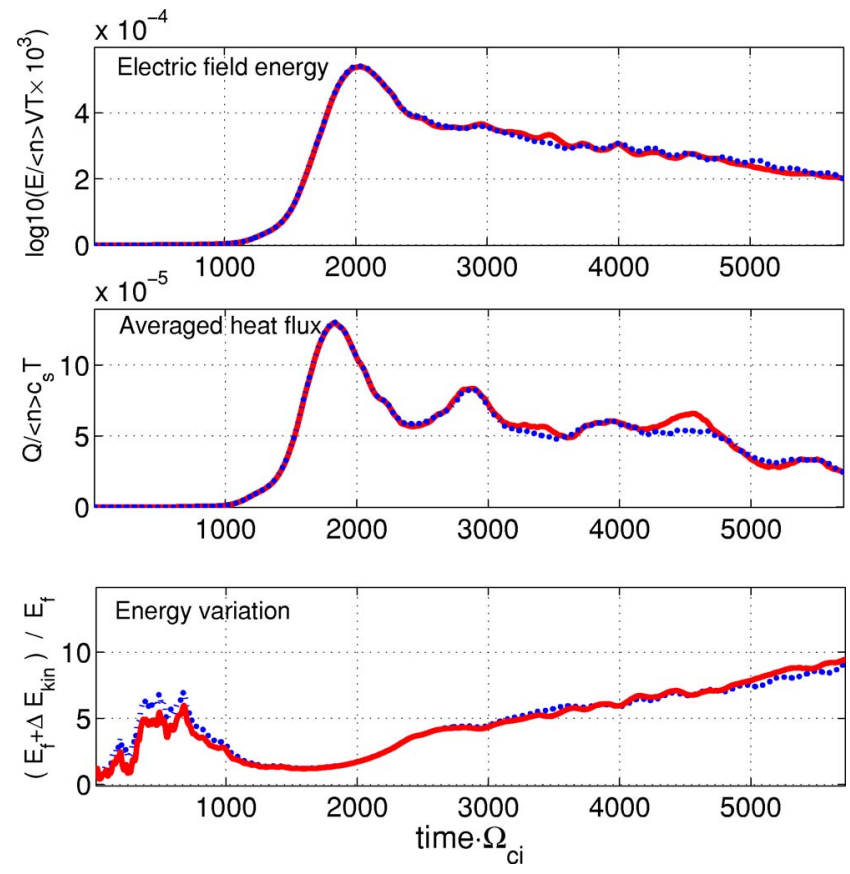

FIG. 8. (Color online): Simulation of toroidal ITG turbulence in the TCV case: standard- $\delta f$ (solid line) versus direct- $\delta f$ (dotted line) scheme. The control variate function is an equilibrium distribution, $f_{0}=f_{\text {eq. }}$. In order to compare the two schemes, we plot the electric field energy, the averaged heat flux and the energy variation. $E_{f}$ is the electric field energy, and $\Delta E_{k i n}$ is the variation of the total particle kinetic energy.

of the result relies directly on the accuracy in the particle trajectory computation. In the standard- $\delta f$ scheme, $\delta f$ is obtained from the integration of Eq. (A11), that we rewrite as

$$
\frac{\mathrm{d}}{\mathrm{d} t} \delta f=-f_{0}\left(\left.\frac{\mathrm{d} \mathbf{Z}}{\mathrm{d} t}\right|_{1} \cdot \nabla \ln f_{0}\right),
$$

$f_{0}$ and its logarithmic derivative in phase space are required to build the right hand side of this equation. In this case, since $f$ and $\delta f$ are known, Eq. (A13) can be used to evaluate $f_{0}$, which now is not directly related to the particle trajectory computation. This means that the two schemes can give numerically different results depending on the accuracy in the particle trajectories computation, which, among other things, relies on the accuracy in the equilibrium reconstruction. Although the distribution function $f_{0}$ is, in general, much larger than $\delta f$, at least at the beginning of the simulation, inaccu- racy in the calculation of $f_{0}$ can lead to spurious values of $\delta f$. For this reason, in order to compare results from the two schemes, $f_{0}$ should be computed in the same way in both and an accurate equilibrium must be supplied. The code ORB5 can run with either scheme. Figure 8 shows a comparison of two simulations that differ only for the $\delta f$ computation method. Since the equations of our model do conserve energy and particle number, ${ }^{7}$ these two quantities (i.e., the corresponding moments of the distribution function) can be used to evaluate the quality of a simulation and the level of numerical noise. The small residual differences in the two simulations come from the fact that an additional potential source of numerical inaccuracies in the standard- $\delta f$ comes from the time integration of the evolution equation, Eq. (A12). So, late in the simulation, conservation properties are slightly improved with the direct- $\delta f$ (see Fig. 8). This is not true at the beginning of the simulation, where the error coming from particle trajectories evaluation can be bigger than the statistical noise. An additional remark: in the standard- $\delta f$ scheme, the sampling of the gradient of $f_{0}$ by markers requires a smaller time step in order to converge. In the direct- $\delta f$ scheme, where $f_{0}$ and not its gradient is sampled, a larger time step is allowed.

It should be noted that both methods are equivalent for any choice of $f_{0}$, provided Eq. (A12) [and not Eq. (A11)] is used in the $\delta f$ scheme, otherwise the results obtained can differ. Using Eq. (A11) for the standard- $\delta f$ scheme requires choosing $f_{0}$ as a gyrokinetic equilibrium, i.e., a function of the constants of unperturbed motion.

${ }^{1}$ P. H. Diamond, S. I. Itoh, K. Itoh, and T. S. Hahm, Plasma Phys. Controlled Fusion 47, R35 (2005).

${ }^{2}$ Y. Idomura, S. Tokuda, and Y. Kishimoto, Nucl. Fusion 43, 234 (2003).

${ }^{3}$ S. E. Parker and W. W. Lee, Phys. Fluids B 5, 77 (1993).

${ }^{4}$ S. Allfrey and R. Hatzky, Comput. Phys. Commun. 154, 98 (2003).

${ }^{5}$ T. M. Tran, K. Appert, M. Fivaz et al., in Theory of Fusion Plasmas, Int. Workshop (Editrice Compositori, SIF, Bologna, 1999).

${ }^{6}$ T. Hahm, Phys. Fluids 31, 2670 (1988).

${ }^{7}$ R. Hatzky, T. M. Tran, A. Könies et al., Phys. Plasmas 9, 898 (2002).

${ }^{8}$ H. Lütjens, A. Bondeson, and O. Sauter, Comput. Phys. Commun. 97, 219 (1996).

${ }^{9}$ Z. Lin, T. Hahm, W. Lee, W. Tang, and R. White, Science 281, 1835 (1998).

${ }^{10}$ A. Y. Aydemir, Phys. Plasmas 1, 822 (1994).

${ }^{11}$ In fact, without collisions, the equilibrium distribution functions of ions and electrons differ, even in a full kinetic model. Therefore, even if the problem here is associated with adiabatic electrons, it has a more extended impact on gyrokinetic simulations. 